\title{
Fabrication of Double-Doped Magnetic Silica Nanospheres and Deposition of Thin Gold Layer
}

\author{
Sang-Eun Park, Jea-Won Lee, Seung-Joo Haam," and Sang-Wha Lee* \\ Dept. of Chemical and Bio Engineering, Kyungwon Lnwersity, Gyeonggi 461-701, Korea. "E-mail: Iswhalaknmgwon.ac.kr \\ Dept of Chemical Engineering, Yonsei Chiversitw, Seoul 120-749. Korea \\ Received Jantary 5, 2009, Accepted Februarv 26, 2009
}

\begin{abstract}
Double-doped magnetic particles that incorporated magnetites into both the surface and inside the silica cores were fabricated via the sol-gel reaction of citrate-stabilized magnetites with silicon alkoxide. Double-doped magnetic particles were easily fabricated and exhibited an higher magnetism in comparison to the singledoped magnetic particles that were prepared by the erosion of surface-deposited magneties from double-doped magentic particles. Thin gold laver was formed over magnetic silica nanospheres via nanoseed-mediated growth of gold clusters. The plasmon-derived absorption bands of double-doped magnetic silica-gold nanoshells were more broadened and shifted down by $c a$. $20 \mathrm{~nm}$ as compared to those of single-doped magnetic silicagold nanoshells, which were attributed to not only the surface scattering of incident light due to relatively rough surafce morphology, but also heterogeneous penmittivity of dielectric cores due to surface-deposited magnetites.
\end{abstract}

Key Words: Double-doped, Magnetites. Gold, Silica. Nanoshell

\section{Introduction}

Nanotechnology, one of the research frontiers in modern science and technology. has focused on the fabrication of hybrid nanostnuctures with unique optical and electromagnetic properties. ${ }^{1.2}$ Gold nanoparticles can be an excellent candidate for biomedical applications owing to their unique optical properties along with biocompatible affinity for various functional ligands. ${ }^{3.4} \mathrm{Halas}$ and co-workers have reported that silica-gold nanoshells (SGNSs) successfully delivered a hyperthermic dose on the destruction of tumor cells via surface plasmon resonance (SPR) that is the collective oscillation of the conduction electrons confined in the metallic interface. ${ }^{5.6}$

The core-shell gold nanostructures combined with magnetic nanoparticles can provide a promising impact on simultaneous diagnostic imaging and targeted hyperthermia effect. In recent years. Diaz and other group reported SGNSs with magnetite-embedded in the silica cores. ${ }^{.8}$ Hyeon and co-worker also prepared silica-gold nanoshells (SGNSs) with magnetiteassembled on the silica cores. ${ }^{9}$ The previous magnetic SGNSs used as a MRI (magnetic resonance imaging) and hy'perthermia agent however exhibited relatively low contents of magnetites in silica matrix. probably due to single-doped magnetites either inside or outside the core. To ameliorate the detection sensitivity of MRI under the deteriorating biological medium. it is necessary to increase the contents of magnetites in the carrier medium or replace higher supermagnetic materials.

Most core-shell nanostructures were fabricated through the complex assembly process of small nanoparticles on ligandfunctionalized oxide cores by electrostatic and/or chemical bonding. For instance. Mirkin et al. and other worker prepared the modified silica nanoparticle assembled with positivelycharged amine groups for the attachment of negativelycharged $\mathrm{Fe}_{3} \mathrm{O}_{4}$ nanoparticles. ${ }^{10.11}$ The water-intoil (W/O) microemulsion has been used to synthesize the uniform magnetic materials. in which micelles (or inverse micelles) are used to control the thickness of silica layer on magnetic nanoparticles. ${ }^{12}$ Core-shell gold composites were also prepared by a multistep (layer-by-layer, LBL) strategy based on the alternative assembly of oppositely-charged polyelectrolytes onto the colloidal templates. 13

In contrast with the complex assembling process for singledoped magnetic nanoparticles. we reported the fabrication of double-doped magnetic silica nanospheres that incorporated magnetites onto both the inside and outside the core materials. Not only was the double-doped magnetic particles simply fabricated. but also exhibited an higher magnetism in comparison to the single-doped ones. ${ }^{-9}$ Thin gold laver was subsequently formed over magnetic silica nanospheres of which optical properties were influenced by surface morphology and heterogeneous dielectric cores. The facile fabrication of double-doped magnetic particles can be potentially applied to multi-functional magnetic sy'stems such as nanoMRI. bio-analytic assays, and magnetic drug delivery. ${ }^{2,14.16}$

\section{Experimental Section}

Synthesis of magnetic silica nanospheres. To synthesize magnetic silica nanosphere. aqueous ferrofluid was prepared according to Massart's method ${ }^{17.18} \mathrm{FeCl}_{3}$ and $\mathrm{FeCl}_{2}(3: 2$ mole ratio) were mixed with $25 \mathrm{~nL}$ of distilled water, and 20 $\mathrm{mL}$ of ammonium hydroxide ( $28 \mathrm{wt} \% \mathrm{NH}_{3}$ ) was added quickly to the chloride mixture under vigorous mechanical (nonmagnetic) stirring at room temperature. A black solid precipitate was immediately formed. The sediment was redispersed by ultrasonification in $50 \mathrm{~mL}$ of distilled water. Sodium citrate was subsequently added to magnetic ferrofluid and refluxed for 1 hour at $70^{\circ} \mathrm{C}$. Then $0.5 \mathrm{~nL}$ of citratestabilized magnetites $(15 \mathrm{~g} / \mathrm{L}) .9 .5 \mathrm{~mL}$ of $\mathrm{H}_{2} \mathrm{O}$. and $1.12 \mathrm{~mL}$ of $\mathrm{NH}_{4} \mathrm{OH}$ were mixed in $40 \mathrm{~mL}$ ethanol. and then $0.5 \mathrm{~mL}$ tetraetlyyl orthosilicate (TEOS) solution was added to a mixed solution under mechanical stirring for $5 \sim 6$ hours. Finally. 


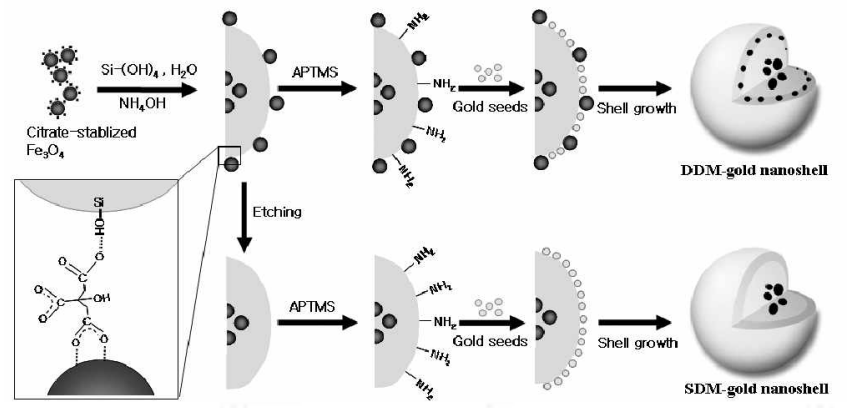

Figure 1. Schematic diagram of synthetic procedures for magnetic gold nanoshells with double-doped and single-doped magnetites.

double-doped magnetic silica nanospheres $\left(\mathrm{Fe}_{3} \mathrm{O}_{4} / \mathrm{aSiO}_{-}-\right.$ $\left(a \mathrm{Fe}_{3} \mathrm{O}_{4}\right.$ ) were obtained by sol-gel reaction of TEOS alkoxide with citrate-stabilized magnetites (CNMPs) in ethanol mixture.

Thin gold layer formation overmagnetic silica nanospheres. To prepare gold colloids of $1 \sim 3 \mathrm{~nm}$. tetrakis(hydroxymethyl) phosphonium chloride (THPC) $/ \mathrm{NaOH}$ reducing agents were mixed with $1.0 \mathrm{wt} \%$ aqueous tetrachlroloaurate(III) trihydrate. ${ }^{19}$ At the alkaline condition. THPC is a powerfully reducing agent enough to reduce gold salts via the derivation of formaldehyde. An excess of 3-aminopropyltrimethoxysilane (APTMS) was then added to a double-doped magnetic silica solution. and APTMS-functionalized magnetic particles were subsequently added to the gold colloids for the deposition of gold seeds onto the surface of magnetic nanoparticles. To form the continuous gold layer over the silica cores. gold-deposited magnetic nanoparticles $\left(\mathrm{Au}_{-} \mathrm{Fe}_{3} \mathrm{O}_{4} a \mathrm{SiO}_{2} a\right.$ $\mathrm{Fe}_{3} \mathrm{O}_{4}$ ) were further reduced by reacting with $\mathrm{K}^{-} \mathrm{AuCl}_{4}{ }^{-}$in the presence of formaldehyde. ${ }^{6}$ Single-doped magnetic silica nanospheres were prepared by the erosion of surface-deposited magnetites in conc. $\mathrm{HCl}$ solution. Thin gold layer was also coated on the single-doped magnetic particles according to the same procedures that were previously described. Figure 1 describes the synthetic scheme for the fabrication of doubledoped and single-doped magnetic silica nanospheres followed by the nanoseed-mediated growth of gold clusters.

\section{Results and Discussion}

$\mathrm{Fe}_{3} \mathrm{O}_{4}$ nanoparticles were prepared by co-precipitation of ferrous and ferric chlorides and the precipitates were processed in ultrasonic dispersion for DLS analysis. The magnetites obtained from the co-precipitation method exhibited a relatively wide distribution of particle sizes. $60 \pm 37 \mathrm{~nm}$. The agglomeration of magnetic ninoparticles provides challenges for them to be a building block for various functional nanocomposites. Thus. it is generally required to modify the surface of magnetites by inorganic and/or organic stabilizers. ${ }^{20.21}$ In our case citrate was employed as a surface-capping agent for $\mathrm{Fe}_{3} \mathrm{O}_{4}$ nanoparticles in order to prevent the agglomeration between magnetites. The size of citrate-capping magnetic nanoparticles (CMNPs) was significantly decreased down to $18 \pm 5 \mathrm{rm}$, which indicated the stabilizing effect of citrate ions on the magnetic particles even though little has been understood about the interfacial coordination of citrate capping. ${ }^{2-}$

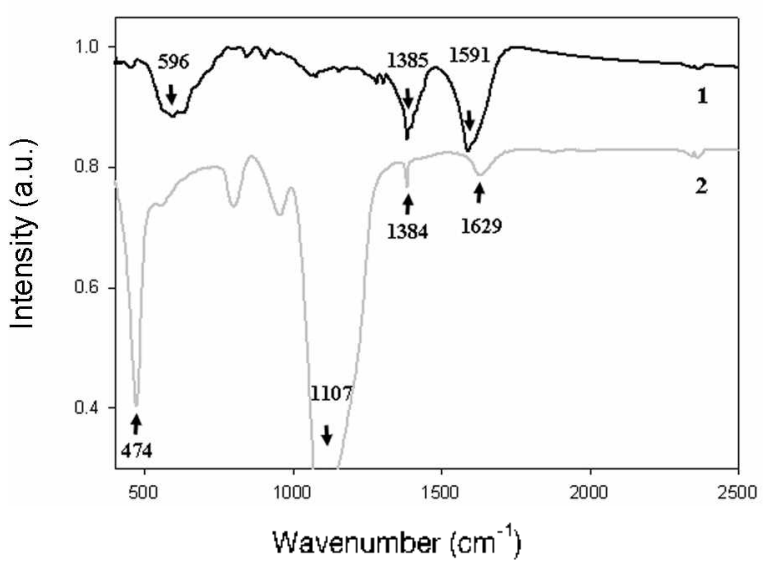

Figure 2. FTIR spectra of citrate-capped magnetites, 1 , and citratecapped magnetites deposited on silica nanospheres, 2 .

In order to elucidate the anchoring effect of citrate-capped magnetites on the silica surface. pristine magnetites and citrate-capped magnetites were added into pure silica colloids prepared by the well-known Stober method. TEM images clearly showed that only citrate-capped magnetites were attached onto silica nanospheres, but there was not any indication of pristine magnetites attached on the silica surface (data not shown). As shown in Figure 2. FTIR spectra of citrate-capped magnetites exhibited the characteristic peak of magnetites at $596 \mathrm{~cm}^{-1}$ and carboxylate groups of citrate at $1385 \mathrm{~cm}^{-1}$ and $1591 \mathrm{~cm}^{-1}$ that were assigned. respectively. to the symmetrical and asymmetrical stretching vibrations. Magnetite-deposited silica nanospheres also exhibited a strong silica peak at $1107 \mathrm{~cm}^{-1}$ and carboxylate groups at $1384 \mathrm{~cm}^{-1}$ and $1629 \mathrm{~cm}^{-1}$, respectively. In contrast with the almost invariant position of symmetrical carboxylate peak. the asymmetrical carboxy late peak in magnetite-deposited silica was distinctly shifted to higher wave numbers by $\mathrm{ca} .38 \mathrm{~cm}^{-1}$ as compared to that of citrate-capped magnetites. The reason may be that that hy'drogen-mediated interaction between citrate carboxylates and silica lyydroxyl groups render a more anisotropic distribution of electron density within magnetitesilica complexes. consequently leading to an upward shift of asy mmetrical stretching bands of carboxylate groups. ${ }^{33: 4}$ At present. it is unclear whether the anchoring effect of citratecapped magnetites is solely due to internnolecular interaction or to ligand exchange. FTIR spectnum analysis. however. suggested that anchoring effect of citrate-capped magnetites was contributed by the hydrogen-bonding interaction between carboxylate groups of citrate and hydroxyl groups of silica surface (see Figure 1).

Double-doped magnetic silica nanospheres $\left(\mathrm{Fe}_{3} \mathrm{O}_{4} \mathrm{OSOO}_{2}-\right.$ $\left(a \mathrm{Fe}_{3} \mathrm{O}_{4}\right)$ were prepared by the modified sol-gel reaction of silicon alkoxide with excessive CMNPs. and single-doped magnetic silica nanospheres $\left(\mathrm{Fe}_{3} \mathrm{O}_{4}\left(a \mathrm{SiO}_{2}\right)\right.$ were obtained by etching the surface-exposed magnetites of double-doped magnetic particles in conc. $\mathrm{HCl}$ solution. As shown in Figure $3(\mathrm{a})$-(b), the surface-deposited magnetites were clearly observed on the surface of silica nanospheres owing to the anchoring effect of citrate carboxylates that was previously 

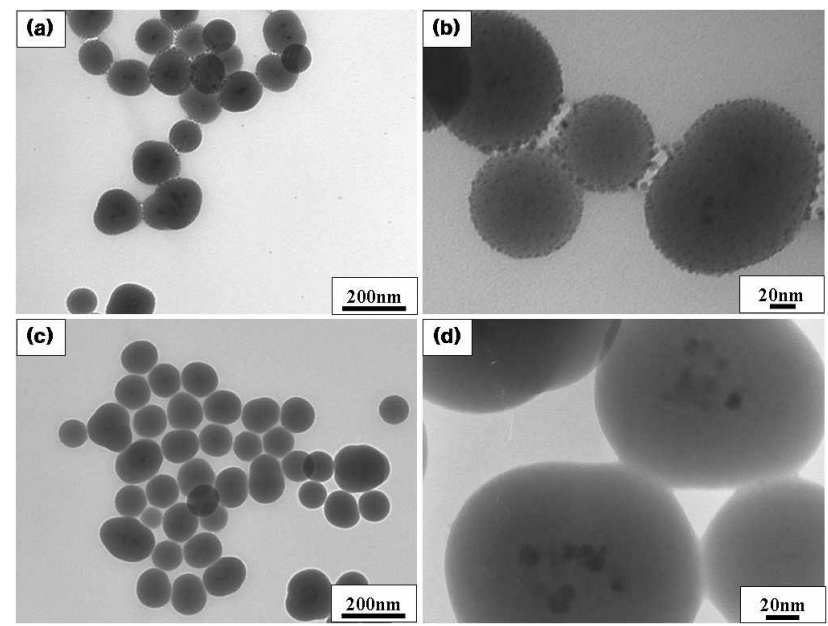

Figure 3. TI:M images of manelite-deposited siliea nanospheres prepared by Stober method of sol-gel teaction: (a) and (b) doubledoped mágnetites (1)] SM), (c) and (d) single-doped magrnetiles (SI)M).

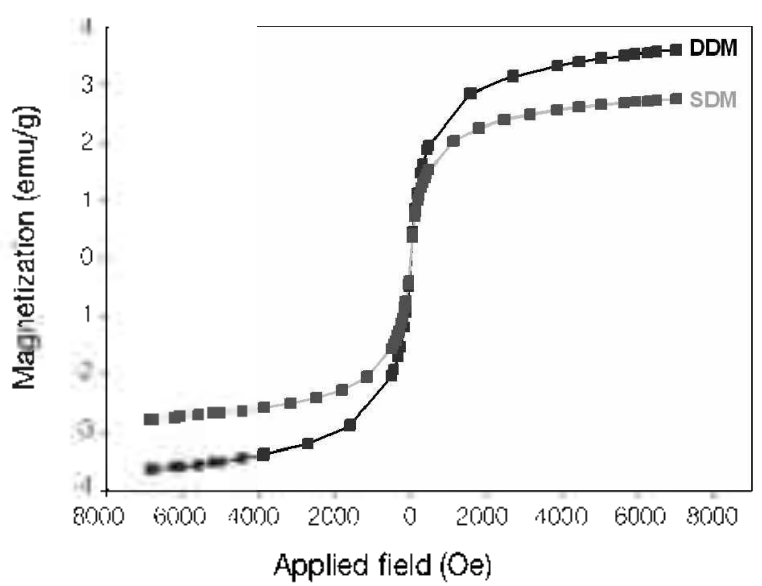

Figure 4. [Jysteresis loops of magnetie silica nanospheres wilh J)I)M and SIM a $300 \mathrm{~K}$, respectively:

described above. As shown in Figure 3(c)-(d). the aggregated magnetites were embedded inside the silica cores during the sol-gel reaction. which seemed to be attributed to the weakened stabilizing effect of deprotonated citratc carboxylates at high alkaline condition. Table I showed that double-doped magnetic (DDM) nanoparticles exhibited higher contents of Fe $w 1 \%$ and magnetism as compared to single-doped magnetic (SDM) nanoparticles, owing to the additional magnetites deposited outside the silica nanospheres. Figure 4 shows the lysteresis loops measured at $1.5 \mathrm{~T}(300 \mathrm{~K})$ of silica nanospheres with double-doped and single-dopod magnetites. Double-doped magnetic (DDM) particles exhibited a higher magnetisn compared to single-doped magnetic (SDM) particles, probably due to the additional magnetites outside the silica nanospheres.

Magnctic silica nanospheres were self-asscmbled by APIMS having terminal amine groups that can give rise to positive reta-potentials. In this case, the reta-potential values were mainly determined by the numbers of amine groups cxposed on magnetic particles. AP'JMS-modified magnetic

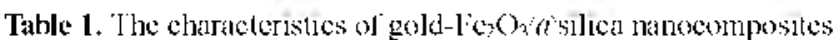

\begin{tabular}{|c|c|c|c|c|}
\hline & \multirow{2}{*}{$\begin{array}{c}\mathrm{F}_{23} \mathrm{O}_{4} \\
\text { máanetites }\end{array}$} & \multirow{2}{*}{$\begin{array}{l}\text { Cilraled-stabi- } \\
\text { lized fieso }\end{array}$} & \multicolumn{2}{|c|}{$\mathrm{I} \mathrm{C}_{3} \mathrm{O}_{1}+\mathrm{SiO}$} \\
\hline & & & 1)IOM & SIMM \\
\hline Size (nm) by DI S ${ }^{i}$ & $60 \pm .37$ & $18 \pm 5$ & $140 \pm 26$ & $114 \pm 25$ \\
\hline Ceta-potential (m V) & -3.3 & -44 & $-57(27)$ & $-43(3.3)$ \\
\hline lew1\%by l:Jux & $-"$ & $-{ }^{c}$ & $2.514 \%$ & $1.13 \times 1 \%$ \\
\hline $\mathrm{em} d / \mathrm{g}_{\mathrm{F}}^{: f}$ & 80 & 46.1 & 3.6 & 2.7 \\
\hline
\end{tabular}

"Size of nanoparticles were determined by intensity -based dynamic laser scallering (1)Is) method in dilute condition of $3 \quad 10^{-1} \mathrm{~m}$ m. 'The reta-potential of each nangrarticles in aqueous solution was measured in the ncutral pH ranges (pH $7 \cdot 8$ ) and bracket number indicates the \%ela-

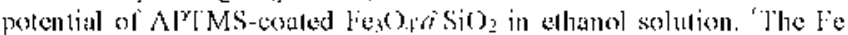
"t" " can be estimated based on the storichiometric commosition of the iron oxide materials. "This represented salurated manchation values by using a vibration sample magnelometer (VSM).
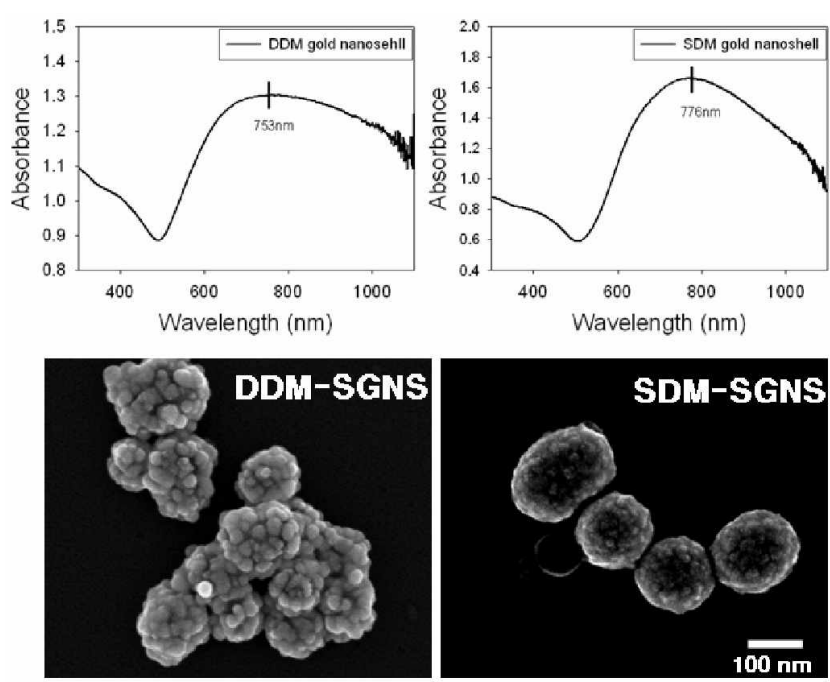

Figure 5. UV-Vis spectra of single-doped magnetic silica-gold nanoshells (SDM-S('NSS) and double-doped manetic siliea-gold nanoshells (1)TMM-S(iNSs) (including S\}:M images). respeetively:

$\mathrm{SiO}_{-}$exhibited the zeta-potential of $+27 \mathrm{mV}$ for double-doped magnetites and $+33 \mathrm{mV}$ for single-doped nagnetites. The low reta-potential values in double-doped magnetic nanoparticles indicated that surface-cxposed magnetites partially serecned the hy droxyl groups of silica nanosphere a ailable for APTMS assembly, consequently reducing amine groups assembled on magnetic silica nanospheres. Au-deposited magnetic nanoparticles were subsequently obtained by the electrostatic attractions between gold colloids with negative surface charges and APTMS-assembled $\mathrm{SiO}_{2}$ with positive surface charges. THPC/ $\mathrm{NaOH}$-induced gold colloids $(\mathrm{I} \sim 3 \mathrm{~nm}$ ) at $\mathrm{ca} . \mathrm{pH} 3.0$ led to monodisperse deposition of gold colloids onto silica nanospheres. ${ }^{25}$ Thin gold laver was formed over magnelic silica nanospheres via secd-mediated growth of gold clusters in the presence of reducible gold salts, $\mathrm{K} \mathrm{AuCl}_{+}$, and formaldehyde reducing agents.

Figure 5 exhibited the SEM images of double-doped magnetic silica-gold nanosholls (DDM-SCiNSs) and singledoped magnctic silica-gold nanoshells (SDM-SGNSs), respectively: The surface morphology of DDM-SGNSs was relatively rough because the coalescence between neigh- 
bouring gold clusters were interfered by surface-deposited magnetites. whereas SDM-SGNS exhibited the smoother surface morphology of thin gold layers. The core radius and shell thickness of SDM-SGNS were determined as ca. $58 \mathrm{~mm}$ and $c a .2 \mathrm{~lm}$. respectively. As shown in Figure 5, the absorption peak of DDM-SGNSs was relatively less intense than that of SDM-SGNSs. Maximal absorption peaks of DDMSGNSs and SDM-SGNSs were positioned at $c a .753 \mathrm{~mm}$ and $776 \mathrm{~nm}$, respectively. Since SPR is a resonant interaction between the electromagnetic field of incident light and the surface charge oscillation. rough surface of metal layer can raise the surface scattering of incident light with the consequent weakening of SPR.

In summary: the plasmon-derived absorption bands of DDM-SGNSs were more broadened and shifted down by $c a$. 20 rum in comparison to those of SDM-SGNS. probably due to the large grain domains of gold clusters and heterogeneous permittivity of dielectric cores attributed to the surfacedeposited magnetites.

\section{Conclusions}

Double-doped magnetic silica-gold nanoshells (DDMSGNSs) were facilely synthesized by the sol-gel process of citrate-stabilized magnetites (CMNPs) with silicon alkoxide. followed by the seed-mediated growth of gold clusters over magnetic silica nanospheres. The successful fabrication of double-doped magnetic silica nanospheres was mainly contributed by two key factors: i) magnetites were stabilized by citrate capping during the sol-gel process, ii) citrate carboxylates capped on magnetites also provided the anchoring effect on the surface of silica nanospheres via hydrogenbonding interaction. Double-doped magnetic particles exhibited higher magnetism in comparison to single-doped magnetic particles. DDM-SGNSs exhibited a relatively rough surface morphology and large grain size owing to the interference of surface-deposited magnetites in the formation of thin gold layer. resulting in the blue-shift of absorption bands and the decrease of absorption intensity. This synthetic route for double-doped magnetic particles can provide a possible multiple-doped magnetic șistem via successive sol-gel reaction of citrate-stabilized magnetites with silicon alkoxide.

Acknowledgments. This work was supported by KOSEF grant funded by the Korea government (MOST) (200605382).

\section{References}

1. Eustis, S.; El-sayed, M. A. Chem. Soc. Rev. 2006, 35, 209.

2. Lu, A. H.; Salabas, E. L.; Schüth, F. Angew. Chem. Int. Ed 2007, t6, 1222

3. Daniel, M. C.: Astruc, D. Chem. Rev. 2004, 104, 293.

4. Brust, M.; Kiely, C. I. Colloids and Surfaces 4. Phusicochemical and Engineening Aspects 2002, 202, 175.

5. Hirsch, L. R.: Stafford, R. I.; Bankson, I. A.; Sershen, S. R.; Rivera, B.: Price, R. E.: Hazle, J. D.: Halas, N. I.: West, I. L. PNAS 2003, 100,13549

6. Pham, T; Jackson, J. B.; Halas, N. J.: Lee, T. R. Langmin 2002, 18,4915

7. Maceira, V. S.; Duarte, M. A. C.; Farle, M.; Quintela, A. L.; Sieradzki, K.; Diaz, R. Chent Hoter, 2006, 18, 2701.

8. Ji, X.; Shao, R.; Elliott, A. M.; Stafford, R. T; Coss, E. E.; Bankson, J. A.; Liang, G.; Luo, Z. P.; Park, K.; Markert, J. T.; Li, C. J. Phvs. Chem. C 2007, 111,6245.

9. Kim, J Park, S.; Lee, J. E.; Jin, S. M.; Lee, J. H.; Lee, I. S.; Yang, I; Kim, J. S.; Kim, S. K, Cho, M. H.; Hyeon, T. Angew. Chem. hit. $E$ d. 2006, 45,7754 .

10. Stoeva, S. I.: Huo, F.: Lee, I. S.: Papaefthymion, G. C.: Kundaliya, D: Ying, T. Y. J. Am. Chem Soc. 2005, 127, 4990.

11. Barnakov, Y. A.; Yu, M. H.: Rosenzweig, Z Langmir 2005, 21, 7524

12. Santra, S:; Tapec, R.; Theodoropoulou, N.; Dobson, I.; Hebard, A.: Tan, W. Langmmir 2001, 17, 2900.

13. Aliev, F. G.: Duárte. M. A. C.: Mamedov, A.: Ostrander. I. W: Giersig, M.: Marzan, L. M. L.: Kotov, N. A. Adv Mater. 1999 , 11. 1006 .

14. Shama, P; Brown, S; Walter, G.; Santra, S.; Moudgil, B. Adv. Colloid hiterface Sci. 2006, 123, 471 .

15. Yang, J.; Lee, C; Ko, H.; Sul, J; Yoon, H.; Lee, K.; Huh, Y.; Haam, S. Angew. Chem. Int Ed. 2007, t6, 8836 .

16. Gupta, A. K.; Gupta, M. Biomaterials 2005, 26, 3995.

17. Massart, R. IEE Trans. I fagn. 1981, 12, 1247.

18. Liu, X : Ma, Z; Xing, T . Liu, H. J. Wagn. Hater, 2004, $270,1$.

19. Duff, D. G.: Baiker, A Langmir 1993, 9, 2310

20. Lu, Y: Yin, Y.: Mayers, B. T.: Xia, Y. Nano Lett. 2002, 2, 183.

21. Li, T.; Deng, Y.; Song, X.; Tin, Z.: Zhang, Y. Bull Korean Chem. Soc. $2003,24,957$.

22. Morais, P. C.: Santos, R. L.; Pimenta, A. C. M.; Azevedo, R. B.; Lima, E. C. D. Thin Solid Film 2006, 515, 266.

23. Hay, M. B.; Myneni, S. C. B. Geochim Cosmochim Acta 2007 , 71,3518 .

24. Lanigan, K. C., Pidsosny, K. Iib. Spectrose $\mathbf{2 0 0 7}, 45,2$.

25. Park, S. E.; Park, M. Y.; Han, P. K.; Lee, S. W. Bull. Konew Chent. Soc. 2006, 27, 1341

26. Barnes, W. L.; Dereux, A; Ebbesen, T. W. Natum 2003, 424 , 824.

27. Raether, H. Surface Plasmons; Springer: Berlin, 1988; p 36.

28. Jung, Y. S.; Sun, Z; Blachere, T.; Kim, H. K. Appl. Phvs. Leth. $2005,87,263116$.

29. Miller, M. M.; Lazarides, A. A. J. Phus. Chem. B 2005, 109 , 21556. 